\title{
Vortex breakdown control in confined swirling flows
}

\author{
RACHID SACI ${ }^{\mathrm{a}}$ AND AMAR KOUADRI \\ Laboratoire Dynamique Des Moteurs et Vibroacoustique, UMB Boumerdes, Algérie
}

Received 5 April 2007, Accepted 9 January 2008

\begin{abstract}
Confined steady flows, which display on-axis or off-axis vortex breakdown, have been numerically studied, with particular attention focused on means of controlling the vortex breakdown bubbles. Firstly, the model flow driven by one end disk of a cylindrical enclosure, which exhibits two distinct on-axis vortex bubbles, has been considered. The resulting computations revealed that, sloping slightly the stationary sidewall, beyond a threshold angle, provided an effective means of removing the secondary vortex structure, and the flow remains steady. Besides, this technique is shown to significantly modify the flow topology which displays off-axis bubbles, induced by the co-rotation of the end disks. Then, the effectiveness of adding a near-axis swirl, as suggested in recent experimental works, has been analysed numerically and found to substantially alter on-axis reverse flows.
\end{abstract}

Key words: Rotating disks / steady flow / vortex breakdown / means of control / numerical simulation

Résumé - Contrôle de l'éclatement tourbillonnaire en écoulement confiné. Cet article propose une étude numérique des écoulements et éclatements tourbillonnaires confinés, stationnaires, avec intérêt particulier réservé aux moyens de contrôle des zones éclatées sur ou détachées de l'axe de rotation. À cet effet, l'étude considère d'abord l'écoulement de base, présentant deux bulbes distincts, engendré par la rotation d'un seul disque d'une enceinte cylindrique. Les résultats révèlent qu'une légère inclinaison de la paroi latérale cylindrique constitue un moyen effectif de suppression des régions éclatées; l'écoulement résultant demeure stationnaire. Par ailleurs, cette approche permet de modifier considérablement la topologie de l'écoulement avec éclatement détaché, engendré par la co-rotation des disques. Enfin, l'influence d'une circulation additionnelle, au voisinage de l'axe, suggérée par des travaux expérimentaux récents, a été analysée et son efficacité confirmée numériquement.

Mots clés : Disques tournants / écoulement stationnaire / éclatement tourbillonnaire / moyens de contrôle / simulation numérique

\section{Introduction}

Swirling flows in cylindrical cavities, driven by the independent rotation of the boundaries, have been the subject of numerous numerical and experimental works, primarily motivated by their widespread engineering applications [1]. Particular interest has been devoted to the phenomenon of vortex breakdown, characterised by an abrupt change in the flow topology, ever since it was observed by Vogel [2], in the model flow driven by the rotation of a single disk of a cylindrical cavity. In this model set up, the flow is governed by only two parameters; namely, the rotational Reynolds number Re and the aspect ratio of the enclosure (height/radius), and characterised by a concentrated vortex core along the axis. Beyond a threshold rotation ratio, the core breaks and

\footnotetext{
${ }^{a}$ Corresponding author: rachid_saci@yahoo.fr
}

gives rise to bubble-like recirculation regions with on axis stagnation points; commonly defined as vortex breakdown [3]. Most subsequent research studies [4-6], aiming at elucidating the fundamental aspects of this feature of the flow, have adopted Vogel's setup, as it presents well defined boundary conditions and provides direct and effective comparison between numerical and experimental simulations.

Escudier [3] carried out extensive experimental investigations and established a detailed flow-regime diagram, based on the variation of the two control parameters: the rotational Reynolds number and the aspect ratio of the enclosure. The diagram, clearly, indicates regions of occurrence of single and multiple (up to three) steady vortex bubbles as well as regions of oscillatory solutions. Escudier [3] also reported that the vortex phenomenon appeared highly axisymmetric; which 


\section{Nomenclature}

\begin{tabular}{|ll|}
\hline & Greek letters: \\
$R e:$ height of the cylindrical container & $\Omega_{\mathrm{b}}:$ angular velocity of the bottom disk \\
$R:$ radius of the cylindrical sidewall & $\Omega_{\mathrm{r}}:$ angular velocity of the rod \\
$R_{\mathrm{b}}$ : bottom disk radius & $\Omega_{\mathrm{t}}:$ angular velocity of the top disk \\
$r_{\mathrm{d}}$ : rod radius & $\nu:$ kinematic viscosity \\
$r_{\mathrm{s}}$ :radial location of the truncated conical sidewall & $\Gamma:$ circulation (angular momentum) \\
$R_{\mathrm{t}}$ : top disk radius & $\xi:$ tangential vorticity component \\
$S:$ rotation ratio of top to bottom disk $\left(S=\Omega_{\mathrm{b}} / \Omega_{\mathrm{t}}\right)$ & $\theta:$ sidewall inclination angle \\
$S_{\mathrm{r}}:$ rod rotation ratio $\left(S_{\mathrm{r}}=\Omega_{\mathrm{r}} / \Omega_{\mathrm{b}}\right)$ & $\psi:$ Stokes's stream function \\
$(r, \theta, z):$ cylindrical coordinates & $\Lambda_{\mathrm{h}}:$ axial aspect ratio $\left(\Lambda_{\mathrm{h}}=H / R_{\mathrm{b}}\right)$ \\
$(u, v, w):$ velocity components & $\Lambda_{\mathrm{r}}:$ radial aspect ratio $\left(\Lambda_{\mathrm{r}}=R_{\mathrm{t}} / R_{\mathrm{b}}\right)$ \\
$t:$ time & $\delta:$ radii ratio parameter $\left(\delta=r_{\mathrm{d}} / R_{\mathrm{b}}\right)$ \\
\hline
\end{tabular}

subsequently motivated extensive 2D numerical investigations, giving accurate steady results comparable with experiments. A noteworthy feature of vortex breakdown, as reported by Tsitverblit [6], is that its onset does not result from a hydrodynamic instability or bifurcation, but appears as a continuous process with increasing Re.

In practical situations, vortex breakdown may be harmful, as observed, for instance, on the tip vortices of a delta-winged aircraft; causing a loss of its control $[1,7]$. On the other hand, it may be beneficial and desired, for example, in bioreactors where it can constitute an ideal environment for cell growth [8]. Moreover, it can enhance mixing in vortex chambers and stabilizes flames in burners [1]. With these considerations in mind, it appeared necessary to investigate appropriate means of vortex breakdown control.

The methods of control reported, in the literature, appear to have been developed and applied, mainly, to bubble-like recirculation regions with stagnation points on the axis, which occur within the concentrated core of the steady model flow, driven by one end wall of a cylindrical enclosure. However, off-axis breakdown induced, for example, in the mid-plane of the flow driven by the same rotation rate of both end walls [9-11]; characterised by a core region in quasi-solid body rotation, appear to have attracted less attention.

In the present work, are explored numerically, intrusive and non-intrusive methods of controlling on-axis as well as off-axis vortex breakdown. These methods are based, essentially, on modifying kinematics and/or geometric conditions upstream of the breakdown. First, we explore the influence of sloping the stationary sidewall on both types of vortex bubbles. Then, we numerically confirm Husain's et al. [7] findings, resulting from an experimental study of the sensitivity of on-axis breakdown to a near-axis swirl, added by means of the differential rotation of a central thin rod. Finally, this later technique has been extended in an attempt to investigate its effects on off-axis vortex breakdown.

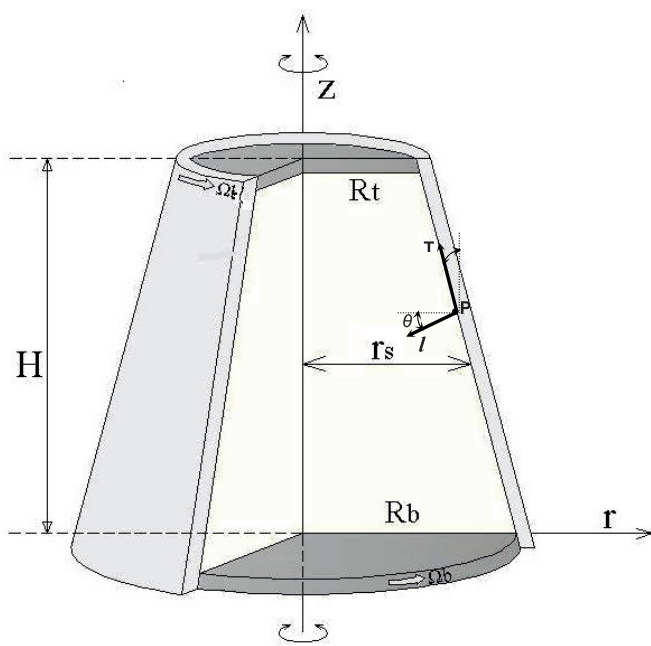

Fig. 1. Schematic truncated conical cavity.

\section{Formulation and numerical approach}

Two distinct configurations, with specific boundary conditions, have been adopted to explore means of vortex breakdown control. So, the corresponding formulations will be presented separately in the following.

\subsection{Truncated conical cavity (Fig. 1)}

Consider the flow in a truncated conical cavity (Fig. 1), driven by the top and bottom end walls of radii $R_{\mathrm{b}}$ and $R_{\mathrm{t}}\left(0<R_{\mathrm{t}} \leqslant R_{\mathrm{b}}\right)$, which rotate with constant, but different, angular velocities $\Omega_{\mathrm{b}}$ and $\Omega_{\mathrm{t}}$, respectively. This configuration introduces a radial aspect ratio parameter which allows the study of the sidewall inclination effects. We note that $R_{\mathrm{b}}=R_{\mathrm{t}}$ corresponds to the particular case of a cylindrical enclosure. Using as the timescale $\Omega^{-1}$ and $R_{\mathrm{b}}$ as a length scale, this configuration introduces the following dimensionless parameters, which govern the dynamics; namely, the Reynolds number, the rotation ratio, the axial and radial aspect ratios, defined respectively by:

$$
R e=R_{\mathrm{b}}^{2} \Omega_{\mathrm{b}} / \nu, S=\Omega_{\mathrm{b}} / \Omega_{\mathrm{t}}, \Lambda_{\mathrm{h}}=H / R_{\mathrm{b}}, \Lambda_{\mathrm{r}}=R_{\mathrm{t}} / R_{\mathrm{b}}
$$


The resulting flow is described using the unsteady axisymmetric Navier-Stokes equations, expressed in cylindrical coordinates and written in a conventional stream function-vorticity formulation. Let $\psi(r, z)$ denote Stokes's stream function, $\Gamma$ the circulation (angular momentum) such that the velocity and the corresponding vorticity fields may be written, respectively:

$$
\begin{aligned}
& (u, v, w)=\left(\frac{1}{r} \frac{\partial \psi}{\partial z}, \frac{\Gamma}{r},-\frac{1}{r} \frac{\partial \psi}{\partial r}\right) \\
& \vec{\nabla} \wedge \vec{V}=\left(-\frac{1}{r} \frac{\partial \Gamma}{\partial z}, \frac{1}{r} \nabla_{*}^{2} \psi, \frac{1}{r} \frac{\partial \Gamma}{\partial r}\right) \\
& \text { where } \nabla_{*}^{2}=\frac{\partial^{2}}{\partial r^{2}}-\frac{1}{r} \frac{\partial}{\partial r}+\frac{\partial^{2}}{\partial z^{2}}
\end{aligned}
$$

The dimensionless transport equations, in terms of the tangential vorticity component $\xi$ and circulation $\Gamma$ are, respectively:

$$
\begin{aligned}
& \frac{\partial \xi}{\partial t}+u \frac{\partial \xi}{\partial r}+w \frac{\partial \xi}{\partial z}-\frac{u \xi}{r}-\frac{2 \Gamma}{r^{3}} \frac{\partial \Gamma}{\partial z}=\frac{1}{R e}\left(\nabla^{2} \xi-\frac{\xi}{r^{2}}\right) \\
& \frac{\partial \Gamma}{\partial t}+u \frac{\partial \Gamma}{\partial r}+w \frac{\partial \Gamma}{\partial z}=\frac{1}{R e}\left(\nabla^{2} \Gamma-\frac{2}{r} \frac{\partial \Gamma}{\partial r}\right)
\end{aligned}
$$

where $\nabla^{2}=\frac{\partial^{2}}{\partial r^{2}}+\frac{1}{r} \frac{\partial}{\partial r}+\frac{\partial^{2}}{\partial z^{2}}$.

The Poisson equation, is written in the form:

$$
\nabla^{2} \psi-\frac{2}{r} \frac{\partial \psi}{\partial r}=r \xi
$$

To solve numerically the above system (2.1)-(2.2), boundary and initial conditions are required. These are based on a no slip condition on the solid walls and an assumption of symmetry on the cavity axis.

\subsubsection{Boundary conditions}

On the bottom rotating disk:

$$
z=0,0<r<1: \quad \psi=0, \Gamma=r^{2}, \quad \xi=\frac{1}{r} \frac{\partial^{2} \psi}{\partial z^{2}}
$$

On the fixed top disk:

$$
z=\Lambda_{\mathrm{h}}, \quad 0<r<\Lambda_{\mathrm{h}}: \quad \psi=0, \quad \Gamma=S \cdot r^{2}, \quad \xi=\frac{1}{r} \frac{\partial^{2} \psi}{\partial z^{2}}
$$

On the cavity axis:

$$
r=0,0 \leqslant z \leqslant \Lambda_{\mathrm{h}}: \quad \psi=0, \Gamma=0, \xi=0
$$

Along the sloped stationary sidewall:

$$
\begin{array}{r}
r=r_{\mathrm{s}}, 0 \leqslant z \leqslant \Lambda_{\mathrm{h}}: \frac{\partial \psi}{\partial l}=0, \quad\left(\frac{\partial^{n} \psi}{\partial T^{n}}\right)_{n \geqslant 1}=0 \\
\Gamma=0, \xi=\frac{1}{r_{\mathrm{s}}} \frac{\partial^{2} \psi}{\partial l^{2}}
\end{array}
$$

The first and the second condition, when $n=1$, on $\psi$ refer to the no-slip and no flow through the sidewall. Here,

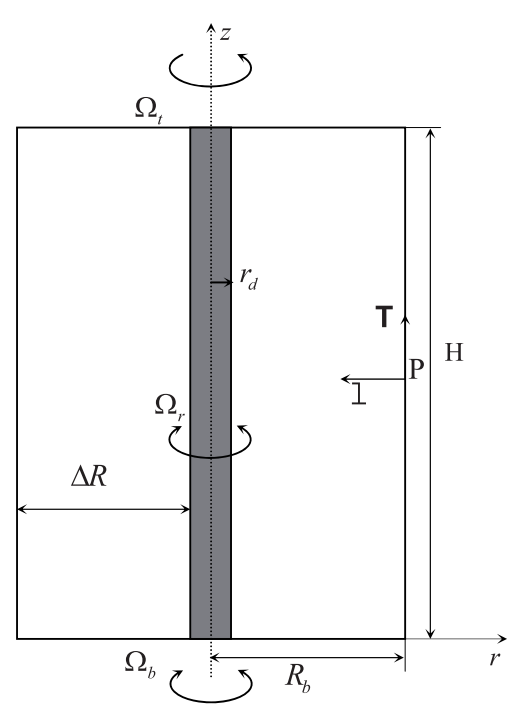

Fig. 2. Schematic cylindrical cavity with central rod.

$(l, T)$ denotes the new coordinate system (Fig. 1 ); $l$ and $T$ being, respectively, the normal and tangential coordinates at a point $\mathrm{P}$ on the sidewall.

The above expressions in (2.6), for the tangential vorticity component along the solid walls, have been derived using the continuity equation in terms of the new coordinates $(l, T)$ and the prescribed conditions of no slip and no flow through the walls. For the computation, $\xi$ is evaluated by expanding out $\psi$ and $\xi$ in Taylor series about a given point on this boundary. More details can be found in references $[12,13]$.

\section{$2.1 .2 \cdot$ Initial conditions}

Let $t<0$ denotes the time when fluid and cavity are at rest. Then, at $t=0$, the top and/or bottom end walls are impulsively rotated with uniform, but different, angular velocities, while the sidewall remains stationary. These are expressed as follows:

$$
t \leqslant 0: \psi=0, \quad \Gamma=0, \xi=0,(r, z) \in] 0, r_{\mathrm{s}}[\times] 0, \Lambda_{\mathrm{h}}[
$$

Here, $r_{\mathrm{s}}$ denotes the radial location of a given point on the sidewall.

$$
t \geqslant 0 \text { : boundary conditions }(2.3)-(2.6) \text { still apply. }
$$

\subsection{Cylindrical cavity with central rod (Fig. 2)}

In this case, we consider a rotor-stator cylindrical cavity with a central thin rod, of radius $r_{\mathrm{d}}$, which can rotate with a uniform angular velocity $\Omega_{\mathrm{r}}$. In this case, the symmetry condition at the axis is replaced by a no slip condition on this rod. When choosing $\Delta R=R_{\mathrm{b}}-r_{\mathrm{d}}$ as a length scale and $\Omega_{\mathrm{b}}^{-1}$ as a time scale, this configuration gives rise to the following control flow parameters:

$R e=\Omega_{\mathrm{b}}(\Delta R)^{2} / \nu, \quad S_{\mathrm{r}}=\Omega_{\mathrm{r}} / \Omega_{\mathrm{b}}, \quad \Lambda_{\mathrm{h}}=H / \Delta R$,

$$
\delta=r_{\mathrm{d}} / R_{\mathrm{b}}
$$


Here, Re denotes the rotational Reynolds number, $S_{\mathrm{r}}$ the rotation rate ratio of the rod and $\delta$ the ratio of the rod radius to that of the bottom disk, which characterises the curvature effect. This later parameter was fixed, throughout the study, in the range $0 \leqslant \delta \leqslant 0.1$ as adopted in previous experimental investigations $[7,14]$. The case $\delta=0$ refers to the cavity with no rod.

The boundary and initial conditions for this second configuration are:

\subsection{1 - Boundary conditions}

On the rotating bottom disk:

$$
z=0, \quad r_{\mathrm{d}}<r<1: \quad \psi=0, \quad \Gamma=r^{2}, \quad \xi=\frac{1}{r} \frac{\partial^{2} \psi}{\partial z^{2}}
$$

On the fixed top disk:

$$
z=\Lambda_{\mathrm{h}}, \quad r_{\mathrm{d}}<r<1: \quad \psi=0, \quad \Gamma=0, \quad \xi=\frac{1}{r} \frac{\partial^{2} \psi}{\partial z^{2}}
$$

On the rotating central rod:

$$
r=r_{\mathrm{d}}, \quad 0 \leqslant z \leqslant \Lambda_{\mathrm{h}}: \quad \psi=0, \quad \Gamma=S_{\mathrm{r}} \cdot r^{2}, \quad \xi=\frac{1}{r_{\mathrm{d}}} \frac{\partial^{2} \psi}{\partial r^{2}}
$$

On the stationary cylindrical sidewall:

$$
r=1, \quad 0 \leqslant z \leqslant \Lambda_{\mathrm{h}}: \quad \psi=0, \quad \Gamma=0, \quad \xi=\frac{1}{r_{\mathrm{d}}} \frac{\partial^{2} \psi}{\partial r^{2}}
$$

\subsection{2 - Initial conditions}

As in the previous case, fluid and cavity are at rest when $t<0$. At $t=0$, the bottom and/or the central rod are impulsively rotated with uniform, but different, angular velocities, while the top and sidewall remain stationary. These conditions take the following form:

$$
t \leqslant 0: \psi=0, \quad \Gamma=0, \xi=0,(r, z) \in] r_{\mathrm{d}}, 1[\times] 0, \Lambda_{\mathrm{h}}[
$$

$t \geqslant 0$ : boundary conditions (2.7)-(2.10) still apply.

To solve the above system of parabolic Equations (2.1) and elliptic Equation (2.2), subject to the prescribed conditions (2.3)-(2.6) or (2.7)-(2.10), we have adopted a three level time-marching finite difference scheme, akin to that employed successfully, in related works, and described in detail by Savvides et al. [12] and by BellamyKnights et al. [13]. Based essentially on numerical experiments, appropriate time and uniform space mesh grids were employed and the time dependent solution is calculated until an ultimate steady state is reached. Poisson's elliptic equation is iterated at each time level, by means of a standard successive over-relaxation method and, subsequently, the azimuthal vorticity component at the boundaries is updated. After a mesh refinement test, most calculations have been carried out on a uniform mesh grid of $100 \times 100 \Lambda_{\mathrm{h}}$ elements. The accuracy of the scheme is first assessed by comparing the present findings, in the case of a cylindrical enclosure, with previous qualitative and

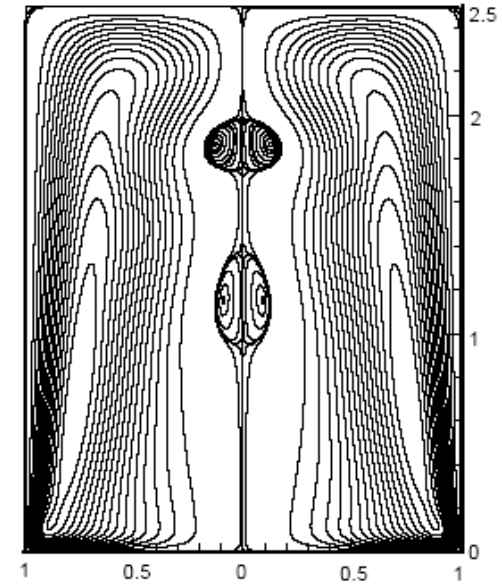

Fig. 3. Meridian streamlines when $R e=2000, S=0, \Lambda_{\mathrm{h}}=$ $2.5, \Lambda_{\mathrm{r}}=1$.

quantitative results reported in the literature by Lopez et al. [11] and Husain et al. [7]. To the authors knowledge, the flow driven by the end walls of a truncated conical cavity does not appear to have been addressed in the literature. In this case, the corresponding results have been assessed, for a couple of flow parameters, by comparing the ultimate converged solution obtained for large times with the solution obtained by solving the steady equations by means of a point successive over-relaxation method. For instance, in the case of the model flow in a rotor stator cavity, described in the next section, for which the asymptotic steady solution was reached approximately when $t=300$, both approaches agreed virtually to within $1 \%$.

\section{Main results}

The investigation reported here, is concerned with steady axisymmetric flow structures. Converged steady solutions were explored in the following range of parameters: $500 \leqslant R e \leqslant 2000,0 \leqslant S \leqslant 1,0.5 \leqslant \Lambda_{\mathrm{r}} \leqslant 1,1 \leqslant$ $\Lambda_{\mathrm{h}} \leqslant 2.5$. The range of the parameter $S_{\mathrm{r}}$, which refers to the differential rotation ratio of the central rod, will be specified as the discussion proceeds.

\subsection{Uncontrolled flow}

\subsubsection{Model flow with on-axis breakdown $(S=0)$}

The steady model flow driven by the bottom end disk of a cylindrical enclosure, of aspect ratio $\Lambda_{\mathrm{h}}=2.5$, is described when $R e=2000$ (Fig. 3). In addition to the azimuthal motion, which the fluid acquires initially at the rotating disk, there develops a secondary circulation with a concentrated central vortex core which breaks to give rise to two distinct on-axis bubbles, characterised by an upstream and a downstream stagnation point $[3,4,15,16]$. 
These are depicted in Figure 3, where meridian streamlines are drawn; non-uniformly spaced so as to emphasize the relatively weak, but relevant, reverse flow regions. Within the disk boundary layer developed initially, angular momentum is advected radially outward. The stationary sidewall causes the fluid to turn into the interior, thus generating a meridian circulation which redistributes the angular momentum. The meridian flow intensifies with increasing $R e$ and, for sufficient inflow towards the axis, a fully developed central vortex core region is generated. Beyond a threshold rotation, the core flow becomes unbalanced: a large radial outward flow is established, causing an axial stagnation point, followed by a reverse flow region.

\subsubsection{Model flow with off-axis breakdown $(S=1)$}

In this case, the end disks of a cylindrical cavity corotate with the same angular velocity $(S=1)$. The resulting steady flow is discussed with reference to Figure 4, when $\Lambda_{\mathrm{h}}=1.7$ and $R e=1700$, which displays a pair of off-axis recirculation bubbles, equally disposed about the mid-plane, as in references [9-11]. The radial location of these features increases with increasing Re. Besides, the flow is characterised by an axial core region in quasisolid body rotation with, virtually, no secondary meridian circulation; the corresponding vortex lines (circulation lines), not reported here, are $z$ independent within this core [11]. The competition of the outward spiralling flows, initially acquired at the rotating disks, combined to the effect of the sidewall confinement, gives rise to a radial inward swirling jet at the mid-depth $z=\Lambda_{\mathrm{h}} / 2$. To further assess our results, we have chosen to illustrate the velocity field which characterises this radial jet flow, as adopted by Lopez et al. [11]. Figure 5, clearly indicates an intensification of the swirl with decreasing radius, attaining a maximum, approximately, on the core surface. We also note that, the radial and azimuthal velocity components are, virtually, of the same order of magnitude outside the core region, which is consistent with Lopez et al. findings [11]. It is also observed, that the radial inflow stagnates $(u=0)$, where the swirl is maximum, causing the divergence of the streamlines and the occurrence of a reverse flow characterising the detached vortex breakdown. From Figures 4 and 5, it is worth noting that the radial extent of the bubble $(u>0)$, at the mid-depth of the enclosure, coincides with the inflexion region of the azimuthal velocity component within the core, which indicates a local break of the quasi-solid rotation within the core.

\subsection{Influence of the sidewall inclination $\left(0.8 \leqslant \Lambda_{r} \leqslant 1\right)$}

\subsubsection{Case when $S=0$}

This part explores the effect of sloping the stationary sidewall of the rotor-stator cylindrical configuration

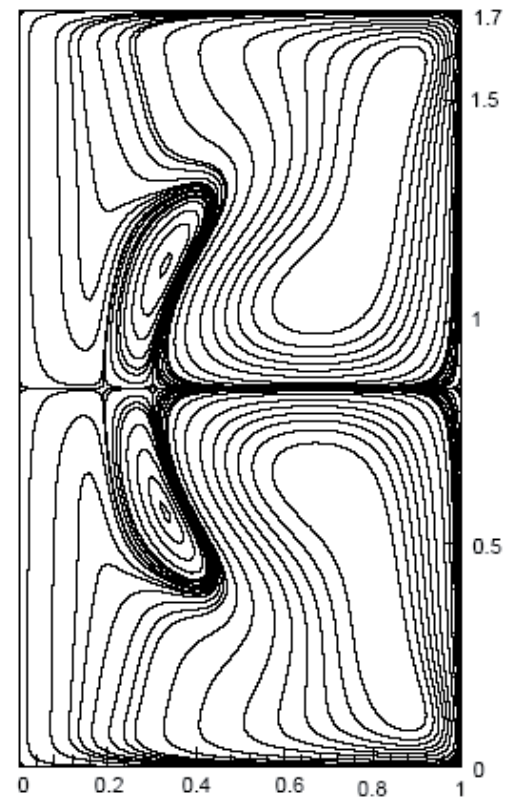

Fig. 4. Meridian streamlines when $R e=1700, S=1, \Lambda_{\mathrm{h}}=$ 1.7, $\Lambda_{\mathrm{r}}=1$.

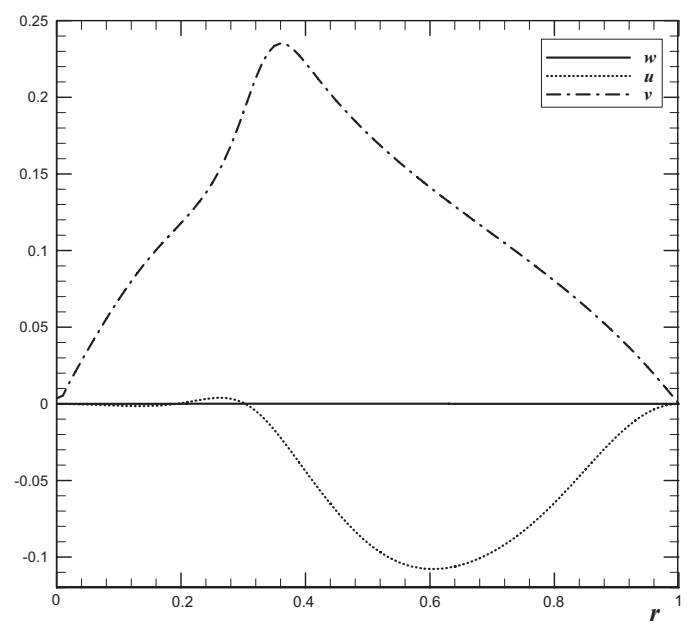

Fig. 5. Velocity profiles at mid-depth $z=\Lambda_{\mathrm{h}} / 2$ when $R e=$ $1700, S=1, \Lambda_{\mathrm{h}}=1.7, \Lambda_{\mathrm{r}}=1$.

discussed in the above section, which, for a given set of parameters exhibits two distinct on-axis bubbles. Aiming to alter the axial swirl upstream the breakdown region, the radial aspect ratio $\Lambda_{\mathrm{r}}$ of the resulting truncated conical cavity is varied in the range $0.8 \leqslant \Lambda_{\mathrm{r}} \leqslant 1$ (which corresponds to an inclination angle $\left(0^{\circ} \leqslant \theta \leqslant 4.57^{\circ}\right)$. We recall that $\Lambda_{\mathrm{r}}=1\left(\theta=0^{\circ}\right)$ corresponds to the cylindrical case (Fig. 6a). The resulting effect is best viewed and described with reference to Figure 6, which illustrates the meridian streamlines corresponding to the model flow driven by the bottom end disk. It is clearly observed that a relatively small perturbation causes large and relevant changes to the vortex structure. In fact, for $\Lambda_{\mathrm{r}}=0.9$ $\left(\theta \sim 2.29^{\circ}\right)$, Figure $6 \mathrm{~b}$ indicates a substantial size reduction of the bubbles, followed by an axially downward shift; 
(a): $\Lambda_{r}=1\left(\theta=0^{\circ}\right)$

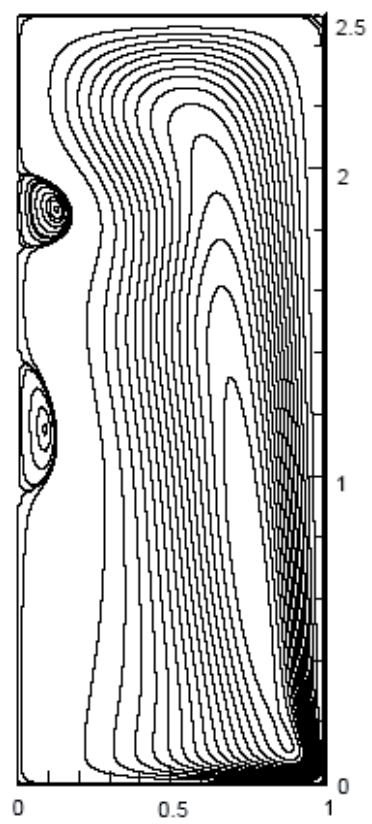

(b): $\Lambda_{r}=0.9\left(\theta \sim 2.29^{\circ}\right)$

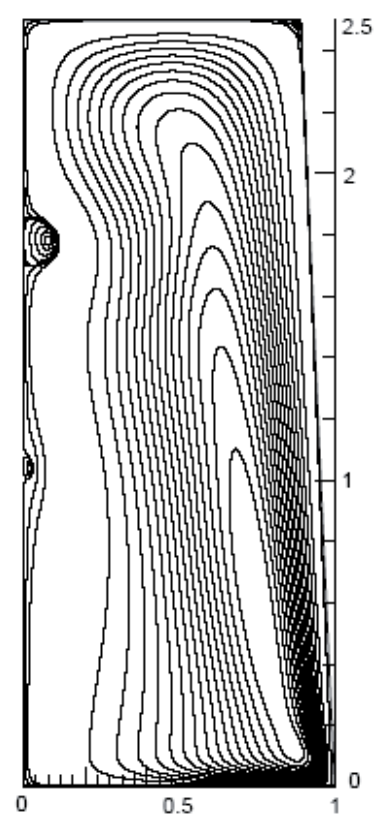

(c): $\Lambda_{r}=0.8\left(\theta \sim 4.57^{\circ}\right)$

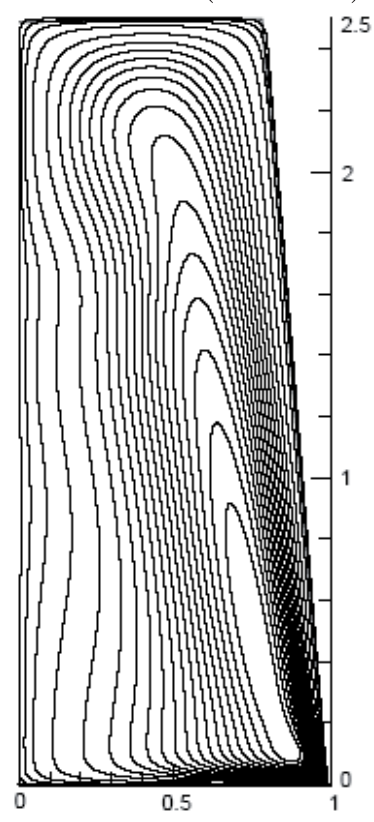

Fig. 6. Meridian streamlines when $R e=2000, S=0, \Lambda_{\mathrm{h}}=$ $2.5 \Lambda_{\mathrm{r}}$ as indicated on each subfigure.

and the threshold value $\Lambda_{\mathrm{r}}=0.8\left(\theta \sim 4.57^{\circ}\right)$, causes the elimination of both vortex breakdown bubbles (Fig. 6c).

Next, we illustrate in Figure 7 how the velocity field $(u, v, w)$ is modified as a result of the sidewall sloping; which led to the suppression of the vortex structures. Figures $7 \mathrm{a}$ and $\mathrm{b}$ show the radial distribution of the velocity components at the axial location $z=1.8$ (axial level of the first bubble), respectively, before and after sloping the sidewall. It is observed that the inclination induces an intensification of the axial downward circulation within
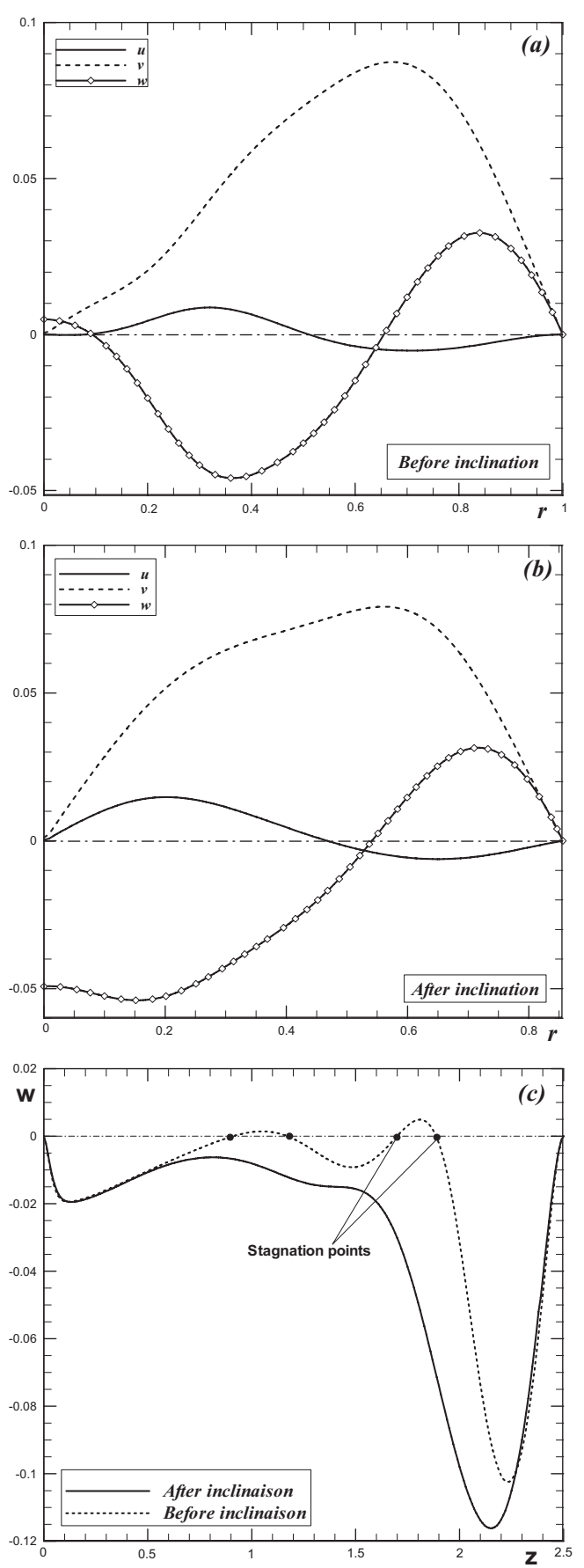

Fig. 7. Velocity profiles before and after he sidewall inclination; when $R e=2000, S=0, \Lambda_{\mathrm{h}}=2.5$; a), b) radial profiles at the axial location $z=1.8$; c) axial velocity distribution at the cavity axis.

the core region which prevents the occurrence of a reverse flow region. This is confirmed by the axial velocity distribution along the cavity axis (Fig. 7c); which clearly indicates an increase in $|w|$, outside the vicinity of the end disks, in the approximate range $0.7 \leqslant z \leqslant 2.25$, leading to an axial downward flow with no on-axis stagnation points $(w<0)$. 

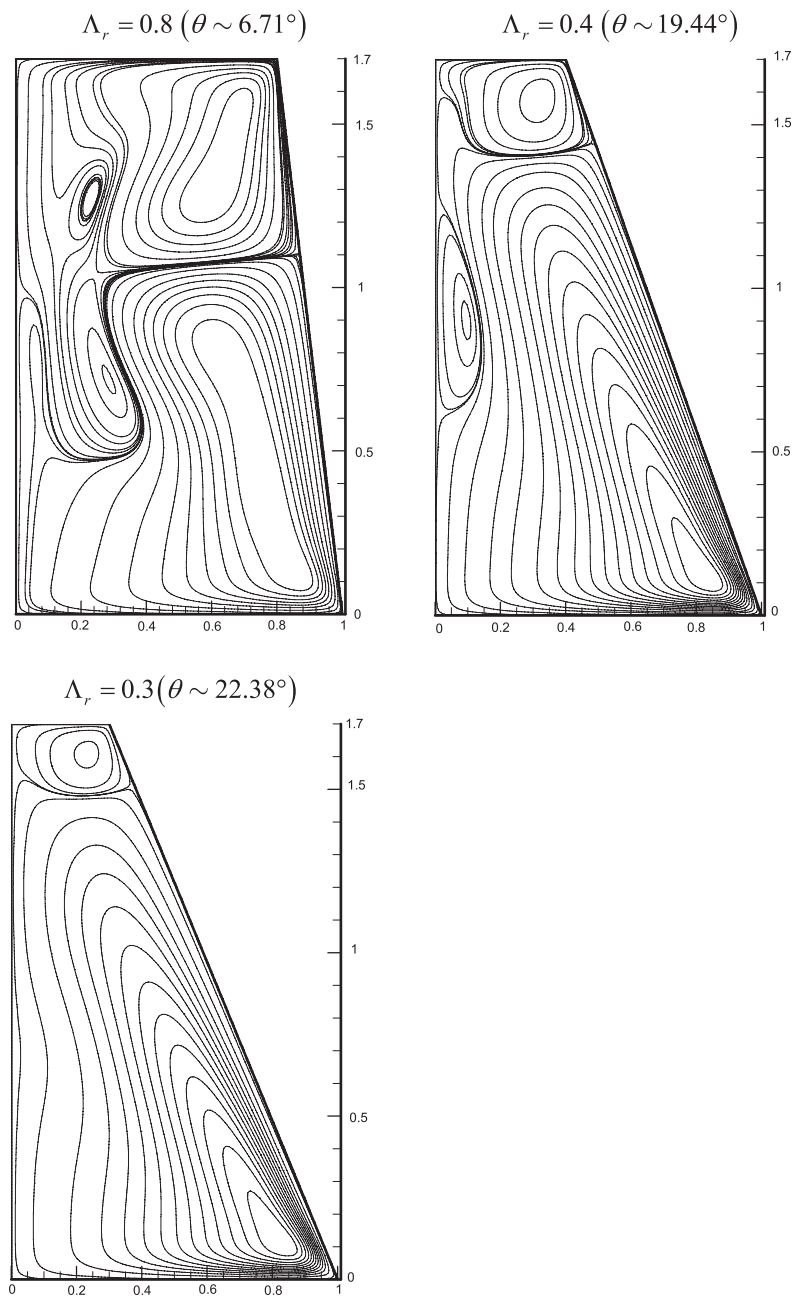

Fig. 8. Meridian streamlines when $R e=1700, S=1, \Lambda_{\mathrm{h}}=$ $1.7 \Lambda_{\mathrm{r}}$ asindicated on each subfigure.

\subsubsection{Case when $S=1$}

The method of control suggested in the first case $(S=0)$ has been extended and applied to investigate the sensitivity of steady off-axis (detached) vortex breakdown, induced by the co-rotation of both end disks of a cylindrical enclosure, with a rotation rate ratio $S=1$. The effect of the sidewall sloping is shown in Figure 8. This figure illustrates meridian streamlines in the case when $R e=1700, S=1, \Lambda_{\mathrm{h}}=1.7$ with a radial aspect ratio in the range: $0.3 \leqslant \Lambda_{\mathrm{r}} \leqslant 0.8$; which corresponds to a sloping angle $6.71^{\circ} \leqslant \theta \leqslant 22.38^{\circ}$. From these selected figures, it is observed that, when $\Lambda_{\mathrm{r}}=0.3$, the inclination causes the suppression of the breakdown phenomenon; leading to a steady flow with no mid-plane symmetry, which displays two simple co-rotating cells, separated by a stagnation line. Besides, when $\Lambda_{\mathrm{r}}=0.4\left(\theta \sim 19.44^{\circ}\right)$, we observe the occurrence of a single vortex bubble located in the core region of the lower dominating cell. (a): without rod
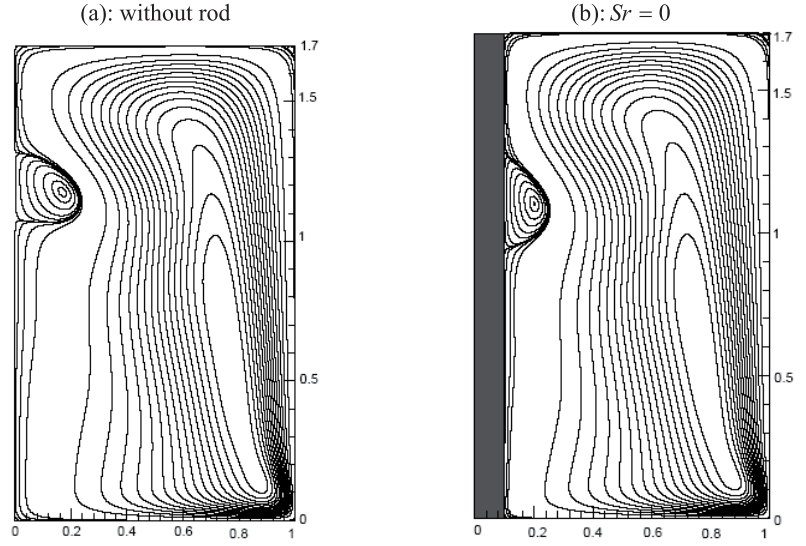

(c): $S r=2$

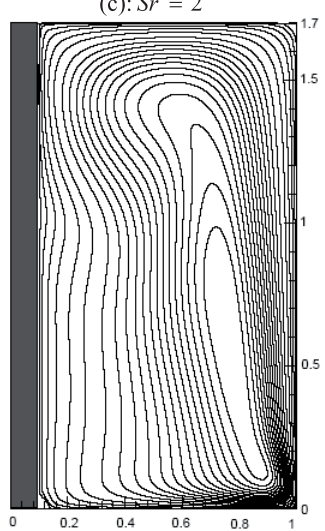

(d): $S r=-2$

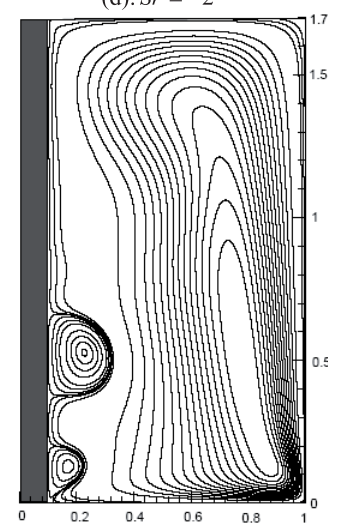

Fig. 9. Meridian streamlines when $R e=1700, S=0, \Lambda_{\mathrm{h}}=$ $1.7, r_{\mathrm{d}}=0.1 S_{\mathrm{r}}$ as indicated on each subfigure.

\subsection{Effect of a near-axis swirl}

\subsubsection{Case when $S=0$}

Previous experimental works [7,14], related to vortex breakdown control in confined swirling flows have introduced a near-axis swirl by means of a rotating central rod mounted at the cylinder axis. Motivated by their experimental findings, the present investigation re-examined and confirmed numerically the effectiveness of this approach to modify the vortex breakdown, which occurs in the concentrated vortex core of the flow driven by only one end wall $(S=0)$.

To illustrate the influence of a differentially rotated thin central rod, on both on-axis and off-axis vortex breakdown, we refer to Figures 9 and 10, respectively, which depict meridian streamlines, in the right half plane, when the rotational Reynolds number $R e=1700$ and the rod radius $r_{\mathrm{d}}=0.1$, in the range of differential rotation rate ratio $-2 \leqslant S_{\mathrm{r}} \leqslant 2$. In this case, the symmetry condition is replaced by a no slip assumption on the rod.

The influence of a differentially rotated thin $\operatorname{rod}\left(S_{\mathrm{r}} \neq\right.$ 0 ) on the on-axis vortex breakdown described above, is viewed with reference to Figure 9 . Figure $9 \mathrm{~b}$ indicates that the presence of a stationary central thin $\operatorname{rod}\left(S_{\mathrm{r}}=0\right)$ has virtually no effect on the vortex structure. In contrast, its co-differential rotation $\left(S_{\mathrm{r}}>0\right)$, which adds a near-axis 

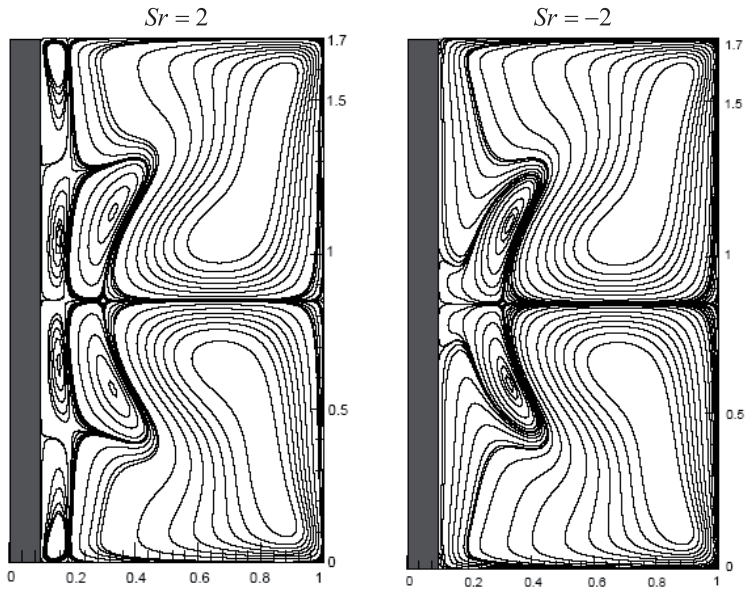

Fig. 10. Meridian streamlines when $R e=1700, S=1, \Lambda_{\mathrm{h}}=$ $1.7, r_{\mathrm{d}}=0.1 S_{\mathrm{r}}$ as indicated on each subfigure.

co-rotating swirl, yields, effectively, to the suppression of the bubbles as clearly depicted in Figure 9c. This result is consistent with Husain's et al. experimental observations [7], carried out using a cylindrical enclosure of aspect ratio $\Lambda_{\mathrm{h}}=3.25$; but contradict Mullin's et al. conclusions [14]; which reported that the rod $\left(0<r_{\mathrm{d}} \leqslant 0.1\right)$ had no qualitative effect on the vortex structure. Moreover, the present investigation, restricted to steady flow features, also revealed that counter-differential rotation $\left(S_{\mathrm{r}}<0\right)$ of the rod, beyond a threshold rate of rotation ratio, increases the bubbles size and induces breakdown enhancement, as shown in Figure 9d, for a rotation ratio $S_{\mathrm{r}}=-2$. As predicted by the experiments [7], it is shown that further increase of the added counter-rotating nearaxis swirl makes the flow unsteady, with more vortex rings along the rod [7].

\subsubsection{Case when $S=1$}

The influence of a rotating central rod on a detached vortex breakdown is shown in Figure 10. Computations revealed that a differential rotation in the approximate range $-2 \leqslant S_{\mathrm{r}} \leqslant 1.7$ has qualitatively no effect on the vortex structure, and the flow remains steady. However, outside this range, the added counter-rotating axial swirl assists the onset of more vortex rings along the rod (Fig. 10), regardless of the direction of rotation. Future work is planed, to explore the transition to time dependent flow regimes and investigate on the stability of the resulting vortex patterns.

\section{Main conclusions}

Methods of controlling vortex breakdown in confined flows, driven by the independent rotation of the end walls of a cylindrical enclosure, have been numerically explored. For on-axis vortex breakdown bubbles with axial stagnation points, generated by the rotation of the bottom end disk, computations revealed that sloping slightly the stationary sidewall of the enclosure constitutes an effective means of removing the vortex structure; the resulting flow remains steady. Besides, this technique has been extended successfully to suppress off-axis-bubbles induced by the co-rotation of the disks, with the same rotation rate. Then, the effectiveness of adding a near-axis swirl, driven by a differentially rotated central thin rod, is analysed and confirmed numerically, in the case of on-axis breakdown. In particular, a differential co-rotation of the rod suppresses the axial bubbles while its differential counterrotation is observed to cause breakdown enhancement. Finally, it is revealed that this approach cannot be extended to suppress off-axis bubbles, regardless of the rate and direction of rotation of the rod.

\section{References}

[1] O. Lucca-Negreo, T. O'Doherty, Vortex breakdown: A review, Prog. Energy Comb. Sci. 27 (2001) 431-481

[2] H.U. Vogel, Experimentelle Ergebnisse über laminar Strömung in einen zylindrischen gehäuse mit darin rotierender scheibe, Phys. Fluids 6 (1968) 2702

[3] M.P. Escudier, Observations of the flow produced in a cylindrical container by a rotating endwall, Exp. Fluids 2 (1984) 189-196

[4] S. Leibovich, The structure of vortex breakdown, Ann. Rev. Fluid Mech. 10 (1978) 221-246

[5] J.M. Lopez, Axisymmetric vortex breakdown part 1. Confined swirling flow, J. Fluid Mech. 221 (1990) 533552

[6] N. Tsitverblit, E. Kit, On the onset of unsteadiness in confined steady flows Fluid Dyn. Res. 23 (1999) 125-152

[7] H.S. Husain, V. Shtern, F. Hussain, Control of vortex breakdown by adding a near-axis swirl, Phys. Fluids 15 (2003) 271-279

[8] L. Manunga, K. Hourigan, M.C. Thompson, Confined flow vortex breakdown control using a small rotating disk, Phys. Fluids 16 (2004) 4750-4753

[9] D.T. Valentine, C.C. Jahnke, Flows induced in a cylinder with both end walls rotating, Phys. Fluids 16 (1994) $2702-2710$

[10] A.Y. Gelfgat, P.Z. Bar-Yoseph, A. Solan, Steady states and oscillatory instability of swirling flow in a cylinder with rotating top and bottom, Phys. Fluids 8 (1996) $2614-2625$

[11] J.M. Lopez, F. Marques, Mode competition between rotating waves in a swirling flow with reflexion symmetry, J. Fluid Mech. 507 (2004) 265-288

[12] C.N. Savvides, J.H. Gerrard, Numerical analysis of flow through a corrugated tube with application to arterial prostheses, J. Fluid Mech. 138 (1984) 129-160

[13] P.G. Bellamy-Knights, R. Saci, Flow between two stationary disks and a rotating shroud, Comput. Fluids 20 (1991) $77-87$

[14] T. Mullin, J.J. Kobine, S.J. Tavener, K.A. Cliffe, On the creation of stagnation points near straight and sloped walls, Phys. Fluids 12 (2000) 425-431

[15] M. Herrada, V. Shtern, Control of vortex breakdown by temperature gradients, Phys. Fluids Rev. 68 (2003) 1-8

[16] M. Piva, E. Meiburg, Steady axisymmetric flow in an open cylindrical container with a partially rotating bottom wall, Phys. Fluids 17 (2005) 1-12 